\title{
A GENERALIZATION OF THE LIGHTBULB THEOREM AND PL I-EQUIVALENCE OF LINKS
}

\author{
RICK LITHERLAND
}

\begin{abstract}
By the "lightbulb theorem" I mean the result that a knot of $S^{1}$ in $S^{1} \times S^{2}$ which meets some $S^{2}$ factor in a single transverse point is isotopic to an $S^{1}$ factor. We prove an analogous result for knots of $S^{n}$ in $S^{n} \times S^{2}$, and apply it to answer a question of Rolfsen concerning PL I-equivalence of links.
\end{abstract}

Introduction. In [9], Rolfsen asked the following

QUESTION. Do there exist links $L=L_{1} \cup \cdots \cup L_{\mu}$ and $L^{\prime}=L_{1}^{\prime} \cup \cdots \cup L_{\mu}^{\prime}$ of $n$-spheres in an $(n+2)$-manifold $M$ such that $L$ and $L^{\prime}$ are I-equivalent and, for each $i=1, \ldots, \mu, L_{i}$ and $L_{i}^{\prime}$ are concordant knots, yet $L$ fails to be concordant to $L^{\prime}$ ?

(This refers to the PL category; I-equivalence is the relation that results when concordances are not required to be locally flat.) The question arises because Theorem 3 of [9] asserts that there are no such links in $S^{n+2}$. The proof of that theorem shows that the answer is no if $n$ is even (since the knot concordance group is zero in even dimensions). We shall show that there are examples for every odd $\boldsymbol{n}$. The case $n=1$ is easily described. We take $M=S^{1} \times S^{2}$. Let $x$ and $y$ be two points of $S^{2}$, set $L_{1}=L_{1}^{\prime}=S^{1} \times\{x\}$ and $L_{2}=S^{1} \times\{y\}$, and let $L_{2}^{\prime}$ be the result of locally tying a trefoil in $L_{2}$. Then $L=L_{1} \cup L_{2}$ and $L^{\prime}=L_{1}^{\prime} \cup L_{2}^{\prime}$ satisfy the desired conditions. In fact $L_{2}$ and $L_{2}^{\prime}$ are ambient isotopic; this is a special case of what is sometimes known as the lightbulb theorem.

To construct examples for greater values of $n$, we would like to replace $S^{1}$ by $S^{n}$ throughout (and the trefoil by some nonslice knot of $S^{n}$ ). To see that this produces links with the right properties, we prove in $\$ 1$ a higher-dimensional version of the lightbulb theorem. For this it seems to be necessary to work in the smooth category. A little triangulation theory gives results for PL case, and hence our examples, in $\$ 2$.

For us, a knot of $M$ in $N$ will mean a submanifold of $N$ isomorphic (i.e., diffeomorphic or PL homeomorphic, as appropriate) to $M$, rather than an embedding of $M$ in $N$. (In the PL case, the submanifold is to be locally flat.) All manifolds will be oriented, and all isomorphisms between manifolds will be orientation preserving. If $M_{1}$ and $M_{2}$ are submanifolds of $N$ and $h: N \rightarrow N$ is an isomorphism, the statement $h\left(M_{1}\right)=M_{2}$ means that $h\left(M_{1}\right)$ and $M_{2}$ are equal as oriented manifolds, i.e., that $h \mid M_{1}: M_{1} \rightarrow M_{2}$ is also orientation preserving.

Received by the editors August 8, 1985.

1980 Mathematics Subject Classification. Primary 57Q45.

(C)1986 American Mathematical Society $0002-9939 / 86 \$ 1.00+\$ .25$ per page 
1. The smooth case. In this section we work in the smooth category. Our aim is to prove

THEOREM 1. Let $\Sigma$ be a smooth knot of $S^{n}$ in $S^{n} \times S^{2}$ such that $\Sigma$ meets some $\{z\} \times S^{2}$ in a single transverse point, and let $\Sigma_{0}=S^{n} \times\{x\}$ for some $x \in S^{2}$. For $n \geqslant 3$, the following are equivalent:

(a) $\Sigma$ is (ambient) isotopic to $\Sigma_{0}$;

(b) $\Sigma$ is ambiently concordant to $\Sigma_{0}$;

(c) $\Sigma$ is concordant to $\Sigma_{0}$;

(d) both of the following hold:

(i) $\Sigma$ is homotopic to $\Sigma_{0}$;

(ii) $i(\Sigma) \subset S^{n+3}$ is unknotted, where $i: S^{n} \times S^{2} \hookrightarrow S^{n+3}$ is the standard inclusion.

If $n=2$ then (b), (c), and (d) are equivalent.

REMARK 1. By ambiently concordant we mean that there is a diffeomorphism $h$ : $S^{n} \times S^{2} \rightarrow S^{n} \times S^{2}$ such that $h\left(\Sigma_{0}\right)=\Sigma$ and $h$ is concordant to the identity.

REMARK 2. The "lightbulb theorem" is the statement that if $n=1$ then (a) always holds (provided that $\Sigma$ is correctly oriented). For $n>1$ this is not the case. If we take $\Sigma$ to be the graph of an essential map $S^{n} \rightarrow S^{2}$ then (d)(i) will not hold. That (d)(ii) may also fail is shown by examples of the kind we need in $\$ 2$, as we now describe.

Let $K$ be a knot of $S^{n}$ in $S^{n+2}$. With $\Sigma_{0}$ as above, define $\Sigma_{K}=\Sigma_{0} \# K$. Then $\Sigma_{K}$ is homotopic to $\Sigma_{0}$, but $i\left(\Sigma_{K}\right)$ is unknotted in $S^{n+3}$ iff $j(K)$ is, where $j$ : $S^{n+2} \rightarrow S^{n+3}$ is the standard inclusion. Let $C^{m, n}$ denote the group of concordance classes of knots of $S^{n}$ in $S^{m}$. According to [4, Theorem 1.2], $C^{m, n}$ is identical with the group of isotopy classes of such knots for $m \geqslant n+3$, so $j(K)$ is unknotted iff $K$ represents an element of the kernel of $j_{*}: C^{n+2, n} \rightarrow C^{n+3, n}$.

Proposition 1. With the notation above

$$
\operatorname{Im} j_{*} \cong \begin{cases}0, & \text { if } n \equiv 0 \bmod 2, \\ 0 \text { or } \mathbf{Z} / 2, & \text { if } n \equiv 1 \bmod 4, \\ \mathbf{Z}, & \text { if } n \equiv 3 \bmod 4,\end{cases}
$$

and $\operatorname{Ker} j_{*}$ is not finitely generated if $n$ is odd.

Proof. Consider first $\operatorname{Im} j_{*}$. For $n \equiv 0 \bmod 2($ respectively $n=1)$ there is nothing to prove, since $C^{n+2, n}=0$ (respectively $C^{4,1}=0$ ). Suppose that $n$ is odd and $n \geqslant 5$. In [7], Levine studies the group $\Theta^{m, n}$ of concordance classes of knots of homotopy $n$-spheres in $S^{m}$; this contains $C^{m, n}$ as a subgroup. According to [4] (see the remark preceding the statement of Corollary 6.6), the image of $j_{*}$ is the intersection of $C^{n+3, n}$ with the kernel of the homomorphism $\omega_{3}(n, 3): \Theta^{n+3, n} \rightarrow \pi_{n}\left(G_{3}, S_{3}\right)$ appearing in Levine's exact sequence (3) ${ }_{3}$; Levine calls this group $\Sigma_{0}^{n+3, n}$. The result now follows from Theorem 6.7 of [7]. 
There remains only the case $n=3$. This is proved in [4, Theorem 5.17] with $C^{5,3}$ and $C^{6,3}$ replaced by the groups of concordance classes of embeddings of $S^{3}$ in $S^{5}$ and $S^{6}$, respectively. However, since $\Gamma_{4}=0$ (Cerf [1]), we obtain the same group from embeddings as from submanifolds.

For the claim about $\operatorname{Ker} j_{*}$ we need to know that $C^{n+2, n}$ is not finitely generated for odd $n$. The knot cobordism groups determined by Levine in [8] coincide with $C^{n+2, n}$ for $n=1$ and $n=3$, and with $\Theta^{n+2, n}$ for other odd $n$. In the latter cases, $C^{n+2, n}$ has finite index in $\Theta^{n+2, n}$ since the group of homotopy $n$-spheres is finite (Kervaire and Milnor [6]).

This shows that (d)(ii) need not be satisfied, at least when $n \equiv 3 \bmod 4$.

Lemma 1. Let $\Sigma$ and $\Sigma_{0}$ be as in Theorem 1. Assume that $\Sigma$ and $\Sigma_{0}$ are homologous. Then there is a diffeomorphism $f: S^{n} \times D^{3} \rightarrow S^{n} \times D^{3}$ such that $f\left(\Sigma_{0}\right)$ $=\Sigma$.

Proof. Let $S^{n}=D_{-}^{n} \cup_{\partial} D_{+}^{n}$, where $z \in$ int $D_{-}^{n}$. We may assume that $\Sigma \cap\left(D_{-}^{n} \times\right.$ $\left.S^{2}\right)=\Sigma_{0} \cap\left(D_{-}^{n} \times S^{2}\right)=D_{-}^{n} \times\{x\}$. Let $\Delta=\Sigma \cap\left(D_{+}^{n} \times S^{2}\right)$ and $\Delta_{0}=\Sigma_{0} \cap\left(D_{+}^{n}\right.$ $\left.\times S^{2}\right)=D_{+}^{n} \times\{x\}$; these are properly embedded $n$-discs in $D_{+}^{n} \times S^{2}$ with $\partial \Delta=\partial \Delta_{0}$. We can also regard them as being contained in $S^{n+2}=D_{+}^{n} \times S^{2} \cup_{\partial} S^{n-1} \times D^{3}$. We claim that there exists a diffeomorphism $g: S^{n+2} \rightarrow S^{n+2}$ such that

(1) $g \mid S^{n-1} \times D^{3}$ is equal to the identity;

(2) $g\left(\Delta_{0}\right)=\Delta$

(3) $g$ is isotopic to the identity.

Now $g$ is defined on $S^{n-1} \times D^{3}$ by (1); we first extend it over $\Delta_{0}$. There is a diffeomorphism $g_{1}: \Sigma_{0} \rightarrow \Sigma$, and we may assume that its restriction to $D_{-}^{n} \times\{x\}$ is the identity since any two orientation-preserving embeddings of the $n$-disc $D_{-}^{n} \times\{x\}$ in $\Sigma$ are isotopic. Extend $g$ by $g_{1} \mid \Delta_{0}$.

Next we claim that $g$ extends over a tubular neighborhood $T_{0}$ of $\Delta_{0}$. The product structure on $\partial\left(D_{+}^{n} \times S^{2}\right)$ gives a normal framing of $\partial \Delta \subset \partial\left(D_{+}^{n} \times S^{2}\right)$, and we are claiming that this extends to a normal framing of $\Delta \subset D_{+}^{n} \times S^{2}$, or equivalently that $\Sigma$ has trivial normal bundle in $S^{n} \times S^{2}$. For $n=2$ this follows from the assumption that $\Sigma$ and $\Sigma_{0}$ are homologous; for $n \neq 2$ any $S_{2}$-bundle over $S^{n}$ is trivial.

At this point, $g$ is defined on $\left(S^{n-1} \times D^{3}\right) \cup T_{0} \cong D^{n+2}$ and is therefore isotopic to the inclusion, allowing us to extend it to all of $S^{n+2}$.

Now regard $S^{n} \times D^{3}$ as $D^{n+3}$ with an $n$-handle attached along $S^{n-1} \times D^{3} \subset$ $S^{n+2}=\partial D^{n+3}$. We obtain $f$ by extending $g$ over $D^{n+3}$ using (3), and over the handle using (1).

The main part of the proof of Theorem 1 is to show that the $f$ provided by Lemma 1 can be chosen so that its restriction to $S^{n} \times S^{2}$ is concordant to the identity. Diffeomorphisms of $S^{p} \times S^{q}$ have been classified up to concordance by Sato [10], for a certain range of values of $p$ and $q$. Unfortunately, this classification is not applicable when $q=2$; we can however use Sato's methods. The following lemma is a consequence of Proposition 1.1 of [10]; we include a proof for the reader's convenience. 
LeMMA 2. Let $f: D^{n+1} \times S^{2} \rightarrow D^{n+1} \times S^{2}$ be a diffeomorphism inducing the identity on $H_{2}\left(D^{n+1} \times S^{2}\right)$, where $n \geqslant 2$. Then the restriction of $f$ to $S^{n} \times S^{2}$ is concordant to the identity.

Proof. Let $k$ be the inclusion of $0 \times S^{2}$ into $D^{n+1} \times S^{2}$. Then $k$ and $f \circ k$ are homotopic, and therefore isotopic by Haefliger [3, Théorème d'existence, (b)]; hence we may assume that $f \mid 0 \times S^{2}=k$. By the tubular neighborhood theorem we may further assume that $f$ maps $\frac{1}{2} D^{n+1} \times S^{2}$ to itself by a bundle isomorphism. Since $\pi_{2}\left(S O_{n+1}\right)=0$ we can arrange that $f \mid \frac{1}{2} D^{n+1} \times S^{2}$ is the identity. Identify $X=$ $\left(D^{n+1}-\operatorname{int}\left(\frac{1}{2} D^{n+1}\right)\right) \times S^{2}$ with $S^{n} \times S^{2} \times I$; then $f \mid X$ is the desired concordance.

Proof of Theorem 1. The implications (a) $\Rightarrow$ (b) $\Rightarrow$ (c) $\Rightarrow$ (d)(i) are trivial. Also (c) implies that $i(\Sigma)$ is null-concordant, and hence (d)(ii) by Theorem 1.2 of [4]. Moreover, (b) $\Rightarrow$ (a) for $n \geqslant 3$ by a theorem of Cerf [2, Corollary 1], so we need only show that (d) $\Rightarrow$ (b) (for $n \geqslant 2$ ). Assume therefore that $\Sigma$ and $\Sigma_{0}$ satisfy (d).

Let $f$ be a diffeomorphism of $S^{n} \times D^{3}$ given by Lemma 1 . Identify $S^{n+3}$ with $S^{n} \times D^{3} \cup_{\partial} D^{n+1} \times S^{2}$ and let $i: S^{n} \times D^{3} \rightarrow S^{n+3}$ be the inclusion. (Thus $i \mid S^{n} \times$ $S^{2}$ is the standard inclusion, previously denoted by $i$.) Since if $\left(S^{n} \times 0\right)$ is isotopic to $i(\Sigma)$, (d)(ii) implies that there is a diffeomorphism $g_{1}$ of $S^{n+3}$, isotopic to the identity, such that $g_{1} f\left(S^{n} \times 0\right)=S^{n} \times 0$. Let $k$ be the diffeomorphism of $S^{n} \times 0$ obtained by restricting $g_{1} f$. Note that $f \circ\left(k^{-1} \times\right.$ id) also satisfies the conclusion of Lemma 1; replacing $f$ by this diffeomorphism we may assume that $g_{1} f \mid S^{n} \times 0$ is the identity. Let $h=f \mid S^{n} \times S^{2}$; the proof will be completed by showing that $h$ is concordant to the identity.

By the tubular neighborhood theorem, there is a diffeomorphism $g_{2}$ of $S^{n+3}$, isotopic to the identity, such that $g_{2} g_{1} f$ maps $S^{n} \times D^{3}$ to itself by a bundle isomorphism. Then $g_{2} g_{1}$ maps $D^{n+1} \times S^{2}$ to itself and induces the identity on $H_{2}\left(D^{n+1} \times S^{2}\right)$ because

$$
\begin{aligned}
\operatorname{Lk}\left(g_{2} g_{1}\left(0 \times S^{2}\right), S^{n} \times 0\right) & =\operatorname{Lk}\left(g_{2} g_{1}\left(0 \times S^{2}\right), g_{2} g_{1} f\left(S^{n} \times 0\right)\right) \\
& =\operatorname{Lk}\left(0 \times S^{2}, f\left(S^{n} \times 0\right)\right) \\
& =\operatorname{Lk}\left(0 \times S^{2}, \Sigma\right) \\
& =\operatorname{Lk}\left(0 \times S^{2}, \Sigma_{0}\right) \\
& =\operatorname{Lk}\left(0 \times S^{2}, S^{n} \times 0\right) .
\end{aligned}
$$

By Lemma 2, $g_{2} g_{1} \mid S^{n} \times S^{2}$ is concordant to the identity. Denote by $C_{0}\left(S^{n} \times S^{2}\right)$ the group of concordance classes of those diffeomorphisms of $S^{n} \times S^{2}$ which induce the identity on $H_{*}\left(S^{n} \times S^{2}\right)$, and let $\alpha: \pi_{n}\left(S_{3}\right) \rightarrow C_{0}\left(S^{n} \times S^{2}\right)$ be the evident homomorphism. We have shown that $h$ represents an element of the image of $\alpha$. Since $\pi_{2}\left(\mathrm{SO}_{3}\right)=0$ we assume from now on that $n \geqslant 3$.

There is a homomorphism $\beta: C_{0}\left(S^{n} \times S^{2}\right) \rightarrow \pi_{n}\left(S^{2}\right)$ which sends the concordance class of a diffeomorphism $g$ to the image of the homotopy class of $g\left(\Sigma_{0}\right)$ under the projection $\pi_{n}\left(S^{n} \times S^{2}\right) \rightarrow \pi_{n}\left(S^{2}\right)$; the condition (d)(i) says that $\beta([h])=0$. 
We have a commutative diagram

$$
\begin{array}{ccc}
\pi_{n}\left(S O_{3}\right) & \stackrel{\alpha}{\rightarrow} & C_{0}\left(S^{n} \times S^{2}\right) \\
\uparrow p_{*} & & \downarrow \beta \\
\pi_{n}\left(S^{3}\right) & \stackrel{H_{*}}{\rightarrow} & \pi_{n}\left(S^{2}\right)
\end{array}
$$

Here $p$ and $\mathrm{H}$ are the double covering $\mathrm{S}^{3} \rightarrow \mathrm{SO}_{3}$ and the Hopf fibration $S^{3} \rightarrow S^{2}$ respectively, and therefore induce isomorphisms on $\pi_{n}$. Hence $h$ is concordant to the identity, as desired.

2. The PL case and I-equivalence of links. In this section all manifolds, homeomorphisms, etc. will be PL unless otherwise stated; in particular, $S^{n}$ will denote the PL $n$-sphere. All submanifolds will be locally flat. Let $M^{n+2}$ be a closed manifold. We denote by $\mathscr{K}(M)(\mathscr{A} \mathscr{C}(M), \mathscr{C}(M))$ the set of ambient isotopy (ambient concordance, concordance) classes of knots of $S^{n}$ in $M$. Suppose that $M_{\alpha}$ is a smoothing of $M$ (i.e., $M_{\alpha}$ is a smooth manifold obtained by giving $M$ a smooth structure such that the identity $M \rightarrow M_{\alpha}$ is a piecewise-differentiable (PD) homeomorphism). By a smooth knot of $S^{n}$ in $M_{\alpha}$ we mean a smooth submanifold $K$ of $M_{\alpha}$ admitting a PD homeomorphism $S^{n} \rightarrow K$; the induced smooth structure on $S^{n}$ is not required to be standard. We denote by $\mathscr{K}\left(M_{\alpha}\right)\left(\mathscr{A} \mathscr{C}\left(M_{\alpha}\right), \mathscr{C}\left(M_{\alpha}\right)\right)$ the set of smooth (ambient) isotopy (ambient concordance, concordance) classes of smooth knots of $S^{n}$ in $M_{\alpha}$.

Let $K$ be a PL knot of $S^{n}$ in $M$ and $K^{*}$ a smooth knot of $S^{n}$ in $M_{\alpha}$. We call $K$ an ambient triangulation of $K^{*}$, and $K^{*}$ an ambient smoothing of $K$, if there is a PD isotopy $H_{t}: M \rightarrow M_{\alpha}$ such that $H_{0}$ is the identity and $H_{1}(K)=K^{*}$. Any smooth knot has an ambient triangulation, and this induces functions $t_{\mathscr{X}}: \mathscr{X}\left(M_{\alpha}\right) \rightarrow \mathscr{X}(M)$ for $\mathscr{X}$ any one of $\mathscr{K}, \mathscr{A} \mathscr{C}$ or $\mathscr{C}$. (This follows from the refinements of Whitehead's triangulation theorems given in [5, Part I, §13].) By Theorem 2 of Wall [11], each $t_{\mathscr{X}}$ is surjective, and (because concordances between knots, being also of codimension 2 , can be ambiently smoothed) $t_{\mathscr{C}}$ is an isomorphism. Denote the standard smoothing of $S^{n}$ by $S_{\text {Diff }}^{n}$.

THEOREM 2. Let $\Sigma$ be a PL knot of $S^{n}$ in $S^{n} \times S^{2}$ such that $\Sigma$ meets some $\{z\} \times S^{2}$ in a single transverse point, and let $\Sigma_{0}=S^{n} \times\{x\}$ for some $x \in S^{2}$. For $n \geqslant 3$, the following are equivalent:

(a) $\Sigma$ is ambient isotopic to $\Sigma_{0}$;

(b) $\Sigma$ is ambiently concordant to $\Sigma_{0}$;

(c) $\Sigma$ is concordant to $\Sigma_{0}$;

(d) both of the following hold:

(i) $\Sigma$ is homotopic in $\Sigma_{0}$;

(ii) $i\left(\Sigma^{*}\right) \subset S_{\text {Diff }}^{n+3}$ is unknotted, where $\Sigma^{*}$ is an ambient smoothing of $\Sigma$ and $i$ is the standard inclusion of $S_{\text {Diff }}^{n} \times S_{\text {Diff }}^{2}$ in $S_{\text {Diff }}^{n+3}$.

If $n=2$ then (b), (c), and (d) are equivalent.

Proof. Since $\Sigma$ can be smoothed by a PD isotopy which is arbitrarily close to the identity in the $C^{1}$-topology, we can take $\Sigma^{*}$ to meet $\{z\} \times S_{\text {Diff }}^{2}$ in a single 
transverse point. The result now follows from the commutative diagram

$$
\begin{array}{ccccc}
\mathscr{K}\left(S_{\text {Diff }}^{n} \times S_{\text {Diff }}^{2}\right) & \rightarrow & \mathscr{A} \mathscr{C}\left(S_{\text {Diff }}^{n} \times S_{\text {Diff }}^{2}\right) & \rightarrow & \mathscr{C}\left(S_{\text {Diff }}^{n} \times S_{\text {Diff }}^{2}\right) \\
\downarrow & & & \downarrow & \\
\mathscr{K}\left(S^{n} \times S^{2}\right) & \rightarrow & \mathscr{A} \mathscr{C}\left(S^{n} \times S^{2}\right) & \rightarrow & \mathscr{C}\left(S^{n} \times S^{2}\right)
\end{array}
$$

and Theorem 1.

Regard $\mathscr{C}\left(S^{n} \times S^{2}\right)$ as a pointed set with basepoint the class of $\Sigma_{0}$. Combining Theorem 2 and Proposition 1 we have

COROLlaRY 1. The kernel of the map $\mathscr{C}\left(S^{n+2}\right) \rightarrow \mathscr{C}\left(S^{n} \times S^{2}\right)$ given by connected sum with $\Sigma_{0}$ is not finitely generated if $n$ is odd.

We can now answer Rolfsen's question.

THEOREM 3. For each odd integer $n$ there are two-component links $L=L_{1} \cup L_{2}$ and $L^{\prime}=L_{1}^{\prime} \cup L_{2}^{\prime}$ of $n$-spheres in $S^{n} \times S^{2}$ such that $L_{i}$ and $L_{i}^{\prime}$ are concordant knots for $i=1,2$ and $L$ is I-equivalent, but not concordant, to $L^{\prime}$.

Proof. For $n>1$, let $K$ be any knot representing a nontrivial element of the kernel of $\mathscr{C}\left(S^{n+2}\right) \rightarrow \mathscr{C}\left(S^{n} \times S^{2}\right)$; for $n=1$ let $K$ be any knot which is not algebraically slice. Let $x$ and $y$ be any two points of $S^{2}$. Let $L_{1}=L_{1}^{\prime}=S^{n} \times\{x\}$, let $L_{2}=S^{n} \times\{y\}$, and let $L_{2}^{\prime}=L_{2} \# K$, so that $L_{2}^{\prime}$ is concordant to $L_{2}$ by choice of $K$ (or by the lightbulb theorem if $n=1$ ). Then $L$ and $L^{\prime}$ are $I$-equivalent; we need to show that they are not concordant. Suppose that $C=C_{1} \cup C_{2} \subset S^{n} \times S^{2}$ $\times I$ is a concordance between them. We can remove a neighborhood of $C_{1}$ and sew it back so as to obtain a manifold $W \supset C_{2}$ with $\partial\left(W, C_{2}\right)=\left(S^{n+2}, 0\right) \sqcup-\left(S^{n+2}, K\right)$. Then $W$ is a homology cobordism so that $K$ is algebraically slice, and hence slice if $n>1$, contrary to assumption. (In fact, if $n>1$ then $W$ is an $h$-cobordism and therefore a product.)

\section{REFERENCES}

1. Jean Cerf, Sur les difféomorphismes de la sphère de dimension trois $\left(\Gamma_{4}=0\right)$, Lecture Notes in Math., vol. 53, Springer, Berlin, Heidelberg, and New York, 1968.

2. The pseudo-isotopy theorem for simply-connected differentiable manifolds, Manifolds-Amsterdam 1970, Lecture Notes in Math., vol. 197, Springer, Berlin, Heidelberg, and New York, 1971, pp. 76-82.

3. André Haefliger, Plongements différentiables de variétés dans variétés, Comment. Math. Helv. 36 (1962), 47-82.

4. __ Differentiable embeddings of $S^{n}$ in $S^{n+q}$ for $q>2$, Ann. of Math. 83 (1966), 402-436.

5. Morris W. Hirsch and Barry Mazur, Smoothings of piecewise linear manifolds, Princeton Univ. Press, Princeton, N. J., and Univ. of Tokyo Press, Tokyo, 1974.

6. Michel A. Kervaire and John W. Milnor, Groups of homotopy spheres: I, Ann. of Math. 77 (1963), 504-537.

7. J. Levine, A classification of differentiable knots, Ann. of Math. 82 (1965), 15-50.

8. K_ Knot cobordism groups in codimension two, Comment. Math. Helv. 44 (1969), 229-244.

9. Dale Rolfsen, Piecewise-linear I-equivalence of links, Low Dimensional Topology (Ed., Roger Fenn), London Math. Soc. Lecture Note Series, vol. 95, Cambridge Univ. Press, Cambridge, 1985, pp. 161-178.

10. H. Sato, Diffeomorphism groups and classification of manifolds, J. Math. Soc. Japan 21 (1969), 1-36.

11. C.T.C. Wall, Locally flat PL submanifolds with codimension two, Proc. Cambridge Philos. Soc. 63 (1967), 5-8.

Department of Mathematics, Louisiana State University, Baton Rouge, Louisiana 70803 